\title{
Mathematical simulation of ammonia gas release in a complex urban terrain using CFD and a statistical approach
}

\author{
M. Kozubková ${ }^{1}$, M. Bojko ${ }^{1}$, O. Zavila ${ }^{2}$, P. Danihelka ${ }^{3}$ \\ \& L. Maléřová ${ }^{2}$ \\ ${ }^{I}$ Department of Hydrodynamics and Hydraulic Equipment, \\ $V \check{S} B$ - Technical University of Ostrava, Czech Republic \\ ${ }^{2}$ Department of Civil Protection, VŠB - Technical University of Ostrava, \\ Czech Republic \\ ${ }^{3}$ Laboratory for Risk Research and Management, \\ VŠB - Technical University of Ostrava, Czech Republic
}

\begin{abstract}
Major accident prevention and preparedness involve the determination of a toxic substance expected or actual release dispersion in the atmosphere, i.e., mathematical modeling of liquid mechanics phenomena. Nowadays, statistical mathematical models are usually used to model simulations of emergency situations in facilities in urban areas or in industrial complexes. Numerical CFD codes have been used mostly for specialized and detailed spatial analyses of physical and chemical phenomena and situations in enclosed spaces. With increasing computing power, these models are beginning to be applied also to complex problems in open spaces, including chemical accidents. Statistical and dynamic models give different results as the principles of the two methods, and the quantity and types of input parameters are different.

The article directly compares the results of simulations of accidental gaseous ammonia releases from an ice arena into a complex urban area, obtained from ALOHA 5.4.3 statistical model and ANSYS Fluent 13.0 numerical CFD model. Real meteorological data were used for the simulations. It emerged that the results of statistical and CFD models may differ radically. The CFD model provided better quality data for addressing accidents.
\end{abstract}


Thanks to the accuracy and comprehensiveness of CFD models, increasing computing performance, and thus reduced time necessary to perform CFD analysis calculations, this approach is increasingly popular in technical and environmental problem solving, although statistical models are still widely used in land-use planning and general emergency preparedness.

Keywords: ALOHA 5.4.3, ANSYS Fluent 13.0, CFD, ammonia, dispersion modeling.

\section{Introduction}

Mathematical modeling of the species dispersion in the atmosphere has been long used to model concentrations or transport of air pollutants emitted from industrial air pollution sources. In chemical accident prevention, it is increasingly often used to model short-term phenomena, i.e., dispersion of toxic gases after their accidental release. The EU Seveso II Directive No. 96/82/ES in Article 12 stipulates the duty to include major accident prevention in land-use planning, and to draw up external emergency plans in Article 11. The new EU Directive 2012/18/EC reiterates the two obligations. Meeting both the requirements is not possible without modeling the extent of toxic effects of escaped pollutants, i.e., mathematical modeling of their dispersion. A similar need to model dispersion after accidental release occurs in other facilities containing toxic substances (ammonia refrigeration or chlorine disinfection systems). Therefore, a question arises concerning suitability of different modeling principles in major accident prevention. The problem was studied by comparing two standard model methods.

The movement and dispersion of ammonia plume, and the reach of ammonia dangerous concentrations after its accidental discharge from an ice arena in a complex urban area was mathematically modeled. A model of a real terrain surrounding the ice arena was created for this purpose and real meteorological input data were used. Ammonia accidental release parameters were based on expert estimates, taking into consideration real arrangement of the ammonia refrigeration system in the ice arena, existing security measures and the amount of gaseous ammonia involved in the accident. The modeling results will be used to optimize the deployment of ammonia detectors installed as a part of the early warning system in the ice arena close proximity, thereby to increase the safety of the public in the vicinity of the object.

\section{Mathematical instruments}

Two mathematical models were used that represented statistical mathematical models and CFD numerical models (see below). In both cases, the models are widely used and generally accepted. 


\subsection{Statistical model - ALOHA software}

Statistical mathematical models were represented by ALOHA 5.4.3 (Areal Locations of Hazardous Atmospheres) [1], an atmospheric dispersion modeling program used for impact evaluation of chemical accidents. The program is noncommercial and freely available on the internet. Some input data are partly preset by means of typical situations of hazardous substance accidental releases or atmospheric stability classes by Pasquill-Giffort. The program can be used to model toxic gas dispersion, fire, or explosion. To calculate toxic gas dispersion the program uses two models: Gaussian and heavy gas. The heavy gas model is used when the molar mass of the dangerous substance is greater than that of the substance (gas) filling the ambient atmosphere (air) or when the temperature of the dangerous substance is lower than the ambient temperature. For all other situations Gaussian model is used. The ALOHA 5.4.3 chooses suitable model itself or the model can be manually selected by the investigator. Modeling results are expressed as iso-lines of dangerous substance concentrations that demarcate 2D map area and determine dangerous zone borders according to the investigator's input.

Statistical models are based on the application of the turbulent diffusion statistical theory. Diffusion of pollutants from a point source is described by a simplified "diffusion equation" that can be solved analytically. Turbulent diffusion follows normal (Gaussian) distribution. The calculation procedure is less time-consuming and costly, but the results for emission source proximities, broken terrains and low flow velocities are unreliable. At zero flow velocity the diffusion equation has no solution.

\subsection{CFD numerical model - ANSYS Fluent software}

CFD (Computational Fluid Dynamics) codes are represented by ANSYS Fluent version 13.0 [4]. It is a sophisticated commercial software tool for fluid mechanics and heat transfer computations. It offers a wide range of sub-models, corresponding input data sets and options. Models included in the software are based on numerical solution of systems of partial differential equations that express the law of conservation of mass (continuity equation), the law of conservation of momentum (Navier-Stokes equations) and the law of conservation of energy (energy equation). This basic set of equations can be supplemented by additional equations that express heat transfer (heat transfer equations - convection, conduction or radiation), or species transport (species transport equations - gas, liquid or solid). The system of equations is then solved with an appropriate numerical method; in this case, with the finite-volume method. The model computes in both 2D and 3D geometries. Calculation results can be visualized as filled or unfilled contours of physical fields, iso-surfaces, animations, 2D value diagrams or numerical data sets.

The continuity equation, Navier-Stokes equations and energy equations are used to calculate the air turbulent flow field. The species transport equation applies to gas pollutant release (e.g., Kozubkova [2], Bojko [3]). 


\subsubsection{Continuity equation for compressible fluid flow}

The continuity equation expresses the law of conservation of mass. For unsteady (time-dependent) compressible fluid flows, it can be written in differential form as

$$
\frac{\partial \rho}{\partial t}+\frac{\partial\left(\rho \cdot \bar{u}_{j}\right)}{\partial x_{j}}=0
$$

where $\rho$ is the fluid density $\left[\mathrm{kg} \cdot \mathrm{m}^{-3}\right], t$ is time $[\mathrm{s}], \bar{u}_{j}$ is the time-averaged $j$ coordinate of the fluid flow velocity $\left[\mathrm{m} \cdot \mathrm{s}^{-1}\right]$, and $x_{j}$ is a coordinate of the Cartesian coordinate system [ - ].

\subsubsection{Navier-stokes equations for compressible fluid flow}

Navier-Stokes equations express the law of conservation of momentum. The substitution of the time-averaged values into the Navier-Stokes equations gives the Reynolds equations. The equation of transfer of momentum for compressible fluids can be written in the form corresponding to differential form as

$$
\begin{gathered}
\frac{\partial\left(\rho \cdot \bar{u}_{i}\right)}{\partial t}+\frac{\partial\left(\rho \cdot \bar{u}_{i} \cdot \bar{u}_{j}\right)}{\partial x_{j}}=-\frac{\partial \bar{p}}{\partial x_{i}}+\frac{\partial}{\partial x_{j}}\left(\mu_{t} \cdot \frac{\partial \bar{u}_{i}}{\partial x_{j}}\right)+\rho \cdot \delta_{i 3} \cdot g \\
+\rho \cdot f_{c} \cdot \varepsilon_{i j 3} \cdot \bar{u}_{j}+\rho \cdot f_{j}
\end{gathered}
$$

where $\rho$ is the fluid density $\left[\mathrm{kg} \cdot \mathrm{m}^{-3}\right], t$ is time $[\mathrm{s}], \bar{u}_{j}$ is the time-averaged $j$ coordinate of the fluid flow velocity $\left[\mathrm{m} . \mathrm{s}^{-1}\right], x_{j}$ is a coordinate of the Cartesian coordinates system [ - ], $\bar{p}$ is the time-averaged value of pressure [Pa], $\mu_{t}$ is the turbulent dynamic viscosity [Pa.s], $\delta_{i 3}$ is the Kronecker delta [- ], $\varepsilon_{i j 3}$ is the unit tensor for centrifugal forces [ - ], $f_{j}$ is the $j$-coordinate of force [N], and $g$ is the gravity acceleration $\left[\mathrm{m} \cdot \mathrm{s}^{-2}\right]$ if buoyancy forces are present.

The equations to express the turbulent flow field variables are turbulent kinetic energy equation $k$ (3) and dissipation rate equation $\varepsilon$ (4). The exact equation for $k$ can be deduced from the Navier-Stokes equations and written as

$$
\begin{gathered}
\frac{\partial k}{\partial t}+\frac{\partial \overline{u_{j}} \cdot k}{\partial x_{j}}=-\frac{\partial}{\partial x_{j}}\left[\overline{u_{j}^{\prime} \cdot\left(\frac{u_{l}^{\prime} \cdot u_{l}^{\prime}}{2}+\delta_{j l} \cdot \frac{p^{\prime}}{\rho}\right)}\right]+v_{t} \cdot \frac{\partial^{2} k}{\partial x_{j}^{2}}-\overline{u_{l}^{\prime} \cdot u_{j}^{\prime}} \cdot \frac{\partial \bar{u}_{l}}{\partial x_{j}} \\
-v \cdot \overline{\frac{\partial u_{l}^{\prime}}{\partial x_{j}} \cdot \frac{\partial u_{l}^{\prime}}{\partial x_{j}}}
\end{gathered}
$$

where $k$ is the turbulent kinetic energy $\left[\mathrm{m}^{2} \cdot \mathrm{s}^{-2}\right], t$ is time $[\mathrm{s}], \bar{u}_{j}$ is timeaveraged $j$-coordinate of the fluid flow velocity $\left[\mathrm{m} . \mathrm{s}^{-1}\right], x_{j}$ is a coordinate of the Cartesian coordinate system [ -$], \rho$ is the fluid density $\left[\mathrm{kg} \cdot \mathrm{m}^{-3}\right], p^{\prime}$ is a 
component of pressure fluctuation $[\mathrm{Pa}]$ and $v_{t}$ is the turbulent kinematic viscosity $\left[\mathrm{m}^{2} \cdot \mathrm{s}^{-1}\right]$.

The turbulent kinetic energy $k$ in eqn. (3) is

$$
k=\frac{1}{2} \cdot\left(\overline{u_{1}^{\prime 2}}+\overline{u_{2}^{\prime 2}}+\overline{u_{3}^{\prime 2}}\right)=\frac{1}{2} \cdot \overline{u_{j}^{\prime 2}}
$$

where $\bar{u}_{j}$ represents time-averaged flow velocity components $\left[\mathrm{m} \cdot \mathrm{s}^{-1}\right]$. The exact equation for $\varepsilon$ can be deduced from the Navier-Stokes equations and written as

$$
\frac{\partial \varepsilon}{\partial t}+\frac{\partial \bar{u}_{j} \cdot \varepsilon}{\partial x_{j}}=\frac{\partial}{\partial x_{j}} \cdot\left(\frac{v_{t}}{\sigma_{\varepsilon}} \cdot \frac{\partial \varepsilon}{\partial x_{j}}\right)+C_{1 \varepsilon} \cdot v_{t} \cdot\left(\frac{\partial \bar{u}_{j}}{\partial x_{l}}+\frac{\partial \bar{u}_{l}}{\partial x_{j}}\right) \cdot \frac{\partial \bar{u}_{l}}{\partial x_{j}}-C_{2 \varepsilon} \cdot \frac{\varepsilon^{2}}{k}
$$

where $\varepsilon$ is the turbulent dissipation rate $\left[\mathrm{m}^{2} \cdot \mathrm{s}^{-3}\right], t$ is time $[\mathrm{s}], \bar{u}_{j}$ is the timeaveraged $j$-coordinate of the fluid flow velocity $\left[\mathrm{m} . \mathrm{s}^{-1}\right], x_{j}$ is a coordinate of the Cartesian coordinate system $[-], v_{t}$ is the turbulent kinematic viscosity $\left[\mathrm{m}^{2} . \mathrm{s}^{-1}\right], \sigma_{\varepsilon}, C_{1 \varepsilon}$ and $C_{2 \varepsilon}$ are empirical constants [- ], and $k$ is the turbulent kinetic energy $\left[\mathrm{m}^{2} \cdot \mathrm{s}^{-2}\right]$. The turbulent kinematic viscosity $v_{t}$ is

$$
v_{t}=C_{v} \cdot \frac{k^{2}}{\varepsilon}
$$

where $C_{v}$ is an empirical constant [- ].

\subsubsection{Energy equation}

The energy equation expresses the law of conservation of energy. According to this law, the change in total energy of the fluid $\bar{E}\left[\mathrm{~J}_{\mathrm{kg}} \mathrm{kg}^{-1}\right]$ in volume $V\left[\mathrm{~m}^{3}\right]$ is determined by the change in the internal energy, kinetic energy, and the flux of both energies through surface $S\left[\mathrm{~m}^{2}\right]$ that surrounds volume $V$. The final equation can be written as

$$
\frac{\partial}{\partial t}[\rho \cdot \bar{E}]+\frac{\partial}{\partial x_{j}}\left[\rho \cdot \bar{u}_{j} \cdot \bar{E}\right]=\rho \cdot \bar{u}_{j} \cdot f_{j}-\frac{\partial\left(p \cdot \bar{u}_{j}\right)}{\partial x_{j}}+\frac{\partial\left(\tau_{j l} \cdot \bar{u}_{j}\right)}{\partial x_{l}}-\frac{\partial \bar{q}_{j}}{\partial x_{j}}
$$

where $t$ is time [s], $\rho$ is the fluid density $\left[\mathrm{kg} \cdot \mathrm{m}^{-3}\right], \bar{E}$ is the time-averaged value of energy $\left[\mathrm{J}_{\mathrm{kg}} \mathrm{kg}^{-1}\right], \bar{u}_{j}$ is the time-averaged $j$-coordinate of the flow field velocity $\left[\mathrm{m} . \mathrm{s}^{-1}\right], x_{j}$ is a coordinate of the Cartesian coordinate system $[-], p$ is the pressure $[\mathrm{Pa}], \tau_{j, l}$ is the tensor of viscous stress $[\mathrm{Pa}]$ and $\bar{q}_{j}$ is the timeaveraged $j$-coordinate of the heat flux $\left[\mathrm{J}^{\mathrm{m}} \mathrm{m}^{-2} \cdot \mathrm{s}^{-1}\right]$. 


\subsubsection{Gas pollutant release equation}

In the model, time-averaged values of the local species mass fraction $\bar{Y}_{i^{\prime}}[-]$ are calculated. These values are described by a balance equation similar to the energy equation (7) that includes both convective and diffuse components of the transport. It can be written in conservative form as

$$
\frac{\partial}{\partial t}\left(\rho \cdot \bar{Y}_{i^{\prime}}\right)+\frac{\partial}{\partial x_{j}} \cdot\left(\rho \cdot \bar{u}_{j} \cdot \bar{Y}_{i^{\prime}}\right)=-\frac{\partial}{\partial x_{i}} \cdot J_{j, i^{\prime}}+R_{i^{\prime}}+S_{i^{\prime}}
$$

where $\bar{u}_{j}$ is the time-averaged $j$-coordinate of the flow field velocity $\left[\mathrm{m} \cdot \mathrm{s}^{-1}\right]$, $R_{i^{\prime}}$ is the production rate of species $i^{\prime}$ due to chemical reaction $\left[\mathrm{kg} \cdot \mathrm{m}^{-3} \cdot \mathrm{s}^{-1}\right]$ and $S_{i^{\prime}}$ is the increment production rate from distributed species $\left[\mathrm{kg} \cdot \mathrm{m}^{-3} \cdot \mathrm{s}^{-1}\right]$. The equation is valid for $N-1$ species, where $N$ is the total number of components included in the mathematical model [ - ]. Species distribution can occur under various conditions. Generally, distribution under laminar and turbulent flow can be distinguished. $J_{j, i^{\prime}}$ represents the diffuse flux of the $i^{\prime}$-component of the mixture $\left[\mathrm{kg} \cdot \mathrm{m}^{-2} \cdot \mathrm{s}^{-1}\right]$. The diffuse flux of the $i^{\prime}$-component in the turbulent flow regime is

$$
J_{i^{\prime}}=-\left(\frac{\mu_{t}}{S c_{t}}\right) \cdot \frac{\partial \bar{Y}_{i^{\prime}}}{\partial x_{j}}
$$

where $\bar{Y}_{i^{\prime}}$ is the time-averaged species $i^{\prime}$ product mass fraction [-] and $S c_{t}$ is the Schmidt turbulent number [ - ] (preset at the default value of 0.7).

\section{Input data}

The input data for calculation with ALOHA 5.4.3 [1] were defined from meteorological data provided by the Czech Hydrometeorological Institute (CHMI). The data represent mean values of meteorological variables measured over the period 2006-2011. The parameters for accidental release (source) of ammonia were based on a qualified estimate derived from documents provided by the ice arena safety engineer. The calculation was performed for wind velocities $2\left[\mathrm{~m} . \mathrm{s}^{-1}\right]$ from the direction $45^{\circ}$ (northeast) and $1.5\left[\mathrm{~m} . \mathrm{s}^{-1}\right]$ from the direction $180^{\circ}$ (south), the temperature in the environment of $0\left[{ }^{\circ} \mathrm{C}\right]$, the atmospheric stability class D (according to Pasquil-Giffort) and the mass flow rate through the ammonia source of $0.586\left[\mathrm{~kg} . \mathrm{s}^{-1}\right]$. A detailed listing of input values for the first scenario above is as follows:

SITE DATA:

Location: OSTRAVA, CZECH REPUBLIC

CHEMICAL DATA:

Chemical Name: AMMONIA

Molecular Weight: $17.03 \mathrm{~g} / \mathrm{mol}$ 
IDLH: 300 ppm

Ambient Boiling Point: $-33.9^{\circ} \mathrm{C}$

Vapor Pressure at Ambient Temperature: greater than 1 atm

Ambient Saturation Concentration: 1,000,000 ppm or 100.0\%

ATMOSPHERIC DATA: (MANUAL INPUT OF DATA)

Wind: 2 meters/second from $45^{\circ}$ true at 10 meters

Ground Roughness: 165 centimeters Cloud Cover: 10 tenths

Air Temperature: $0^{\circ} \mathrm{C} \quad$ Stability Class: $D$

No Inversion Height Relative Humidity: $50 \%$

SOURCE STRENGTH:

Direct Source: 0.586 kilograms/sec Source Height: 6 meters

Release Duration: 10 minutes

Release Rate: 35.2 kilograms/min

Total Amount Released: 352 kilograms

THREAT ZONE: (GAUSSIAN SELECTED)

Model Run: Gaussian

Red: 313 meters --- $(150 \mathrm{ppm}=$ ERPG-2)

Orange: 729 meters --- $(30 \mathrm{ppm}=P A C-1)$

The input data for calculation with ANSYS Fluent 13.0 [4] were also defined from meteorological data provided by the Czech Hydrometeorological Institute (CHMI) [5]. The parameters for accidental release (source) of ammonia were also based on a qualified estimate derived from documents provided by the ice arena safety engineer.

A cuboid-shaped geometry with dimensions of 800 [m] (width) x 800 [m] (length) x $150[\mathrm{~m}]$ (height) was created with the DesignModeler [4] program according to maps obtained from the Cadastral Office of Ostrava (Czech Republic) [6]. The entire geometry was then divided into two cuboids lying on each other. The first cuboid was of dimensions 800 [m] (width) x 800 [m] (length) x $50[\mathrm{~m}]$ (height) and represented a zone close to the earth's surface, i.e., urban area. The second cuboid was of dimensions 800 [m] (width) x 800 [m] (length) x 100 [m] (height) and represented free atmosphere with no interference caused by buildings. The grid of the geometry was created with ANSYS Meshing [4] program. The total number of grid cells was approximately 1.6 million.

The boundary conditions for the geometry were defined by atmospheric pressure of $101325[\mathrm{~Pa}]$, the wind speed, the turbulent kinetic energy, the turbulent dissipation velocity and the air temperature profiles. The wind speed profile was defined as

$$
v=v_{r e f} \cdot\left(\frac{z}{10}\right)^{p}
$$

where $v$ is the air flow velocity $\left[\mathrm{m} \cdot \mathrm{s}^{-1}\right]$ in height $z[\mathrm{~m}], v_{\text {ref }}$ is the referential air flow velocity in height $z=10[\mathrm{~m}]$ and $p$ is the exponent for the atmospheric stability class D (according to Pasquil-Giffort). The referential air flow velocities 
$v_{\text {ref }}$ were $2\left[\mathrm{~m} . \mathrm{s}^{-1}\right]$ for the wind from direction $45^{\circ}$ (northeast) and $1.5\left[\mathrm{~m} . \mathrm{s}^{-1}\right]$ for the wind from direction $180^{\circ}$ (south). The turbulent kinetic energy profile was defined as

$$
k=\frac{v_{*}^{2}}{0.3}
$$

where $k$ is the turbulent kinetic energy $\left[\mathrm{m}^{2} \cdot \mathrm{s}^{-2}\right]$ and $v_{*}$ is the shear velocity, for which the value of $0.4[\mathrm{~m} / \mathrm{s}]$ was selected. The turbulent dissipation velocity profile was defined as

$$
\varepsilon=\frac{v_{*}^{3}}{0.4 \cdot z}, \quad v_{*}=\frac{0.419 \cdot v_{r e f}}{\ln \left(\frac{10+z_{r e f}}{z_{r e f}}\right)}
$$

where $\varepsilon$ is the turbulent dissipation velocity $\left[\mathrm{m}^{2} \cdot \mathrm{s}^{-3}\right], v_{*}$ is the shear velocity $\left[\mathrm{m} . \mathrm{s}^{-1}\right], v_{r e f}$ is the referential air flow velocity in height $z_{r e f}=10[\mathrm{~m}]$. The air temperature profile was defined as

$$
T=T_{0}+273.15+\gamma \cdot z
$$

where $T$ is the temperature $[\mathrm{K}], T_{0}$ is the average temperature $[\mathrm{K}]$ according to CHMI, $\gamma=-0.0065\left[\mathrm{~K} . \mathrm{m}^{-1}\right]$ is the dry adiabatic lapse rate for atmospheric stability class $\mathrm{D}$ and $z$ is the height coordinate $[\mathrm{m}]$. The average temperature $T_{0}$ was $273.05[\mathrm{~K}]=-0.1\left[{ }^{\circ} \mathrm{C}\right]$.

The ammonia source boundary conditions were defined by the source temperature $256.6[\mathrm{~K}]=-16.55\left[{ }^{\circ} \mathrm{C}\right]$ and the mass flow $1.58487\left[\mathrm{~kg} . \mathrm{s}^{-1}\right]$. The pollutant was defined as a mixture of air (mass fraction 0.63 [ - ]) and gas ammonia (mass fraction 0.37 [ - ]) escaping from a surface source with dimensions of 1.4 [m] (width) x 2.4 [m] (length) located at the height of 6 [m] above the ground.

First, the calculation of the turbulent airflow field was performed as stationary (time-independent) task. Then, the source of gas pollutant was activated in the model and the task was completed as non-stationary (time-dependent). The air flow field was modeled using the RNG k- $\varepsilon$ model of turbulence and Species transport model [4] was used for species motion and dispersion modeling. For the purpose of the analysis, the CFD ANSYS Fluent 13.0 software had been verified in a wind tunnel (e.g., Zelinger [8]) on a gas motion and dispersion task.

\section{Results}

The ALOHA 5.4.3 results were exported in KML-format for Google Earth [7] and implemented into aerial maps of the target area as iso-lines of gas ammonia (see Figure 1). The iso-line represents the thread zone border for PAC-1 acute 
toxicity threshold (gas ammonia concentration of 30 [ppm]) and the thread zone border for ERPG-2 acute toxicity threshold (150 [ppm]). The situation was evaluated 10 minutes after the beginning of the accidental release.

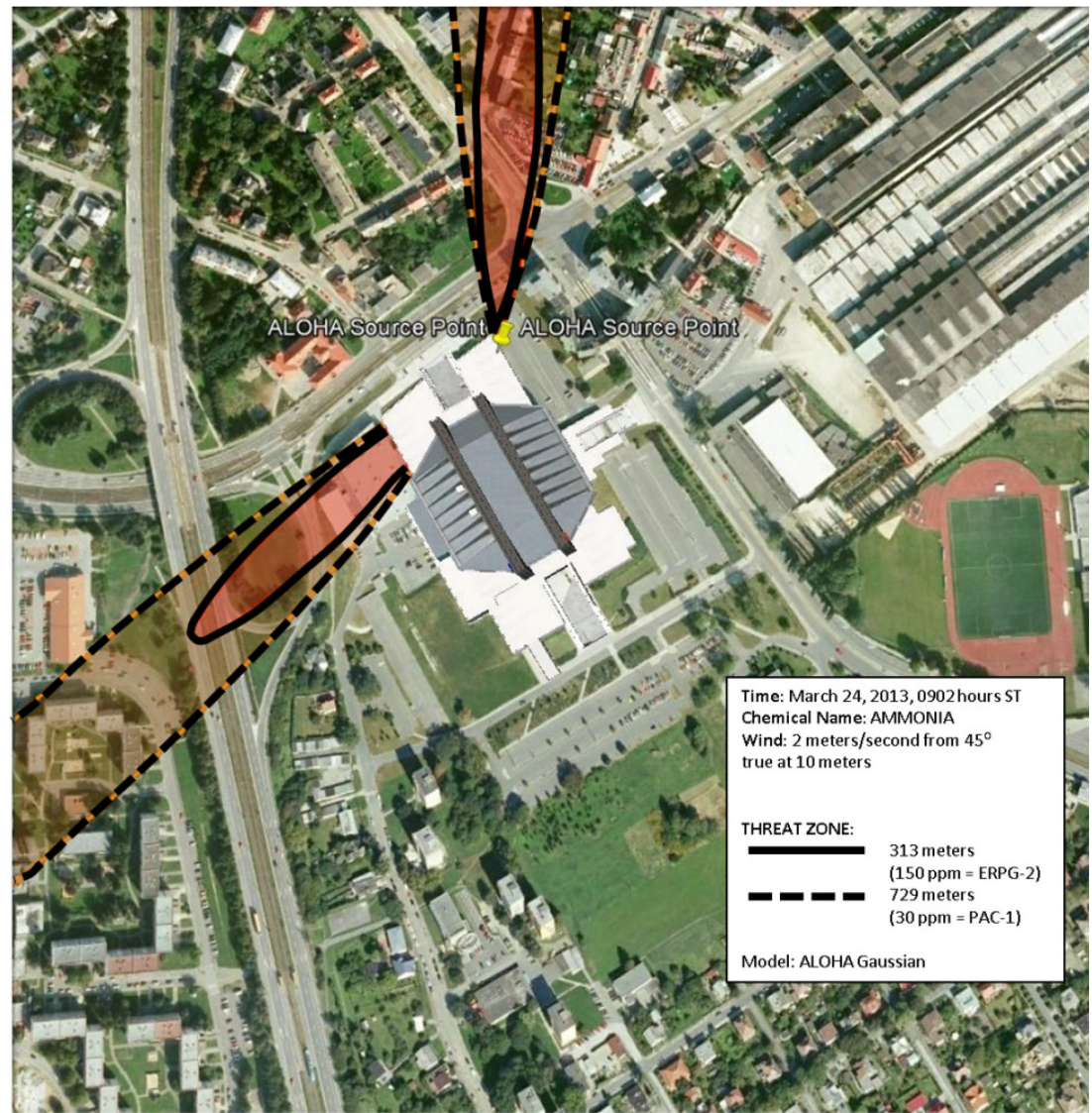

Figure 1: Gas ammonia thread zones modeled with ALOHA 5.4.3.

The obtained results are probably overestimated for the following reasons: In ALOHA 5.4.3, gas mixtures cannot be defined, or rather, the mix components cannot be separately evaluated. It is therefore necessary to define only proportional quantity of fresh medium in the source, which in turn affects its further dispersion. Besides, the specific impact of buildings on the pollutant plume diffusion and dispersion cannot be modeled.

The ANSYS Fluent 13.0 results were visualized as contours of species (ammonia) concentrations in two-dimensional cut planes of three-dimensional geometry at a height of $1.5 \mathrm{~m}$ above the ground (breathing zone). PAC-1 and ERPG 2 acute toxicity thresholds were again used for evaluation. Unlike ALOHA 5.4.3, ANSYS Fluent 13.0 makes possible to define gas mixtures and observe the mix components separately. It also contains turbulence models that 
allow for the effects of mechanical turbulence from surrounding buildings on the motion and diffusion of the gas pollutant plume (see Figure 2). The differences between the two results are obvious. The same study was performed for eight cardinal directions and two seasons. Due to limited space, only one example is presented in this article.

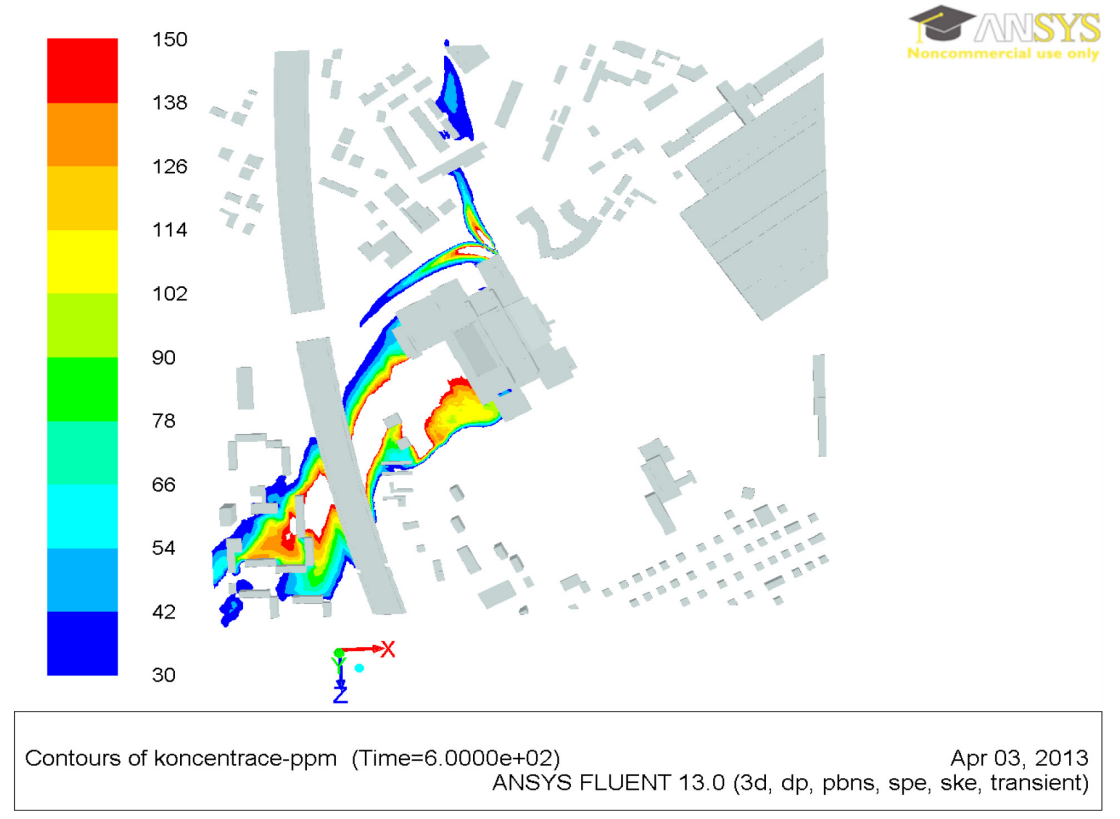

Figure 2: $\quad$ Gas ammonia thread zones modeled with ANSYS Fluent 13.0.

Comparison of the results and mathematical bases of the statistical and the CFD model showed that the statistical model outputs for different directions, that is, under different conditions of the flowed around objects, differ only slightly in a complex urban area, even with real meteorological data. On the other hand, the CFD model shows great differences in distances and spatial distribution of gas concentrations, including iso-lines of endpoints, for different directions of the air flow. Figure 1 and Figure 2 illustrate two variants of the example (two wind directions at different air flow velocities). It also emerged that in contrast to statistical models where the highest concentrations are always found in immediate vicinity of the source, the CFD model was capable to capture situations where the flow around obstacles cause dangerous concentrations to be found farther away from the source (depending on the terrain and buildings).

\section{Discussion}

Comparison of statistical and CFD models showed significant differences between the results of the two models. It also showed differences between the 
results of CFD modeling for different directions, that is, in different terrains. This is the factor that most statistical models cannot allow for. CFD models offer higher accuracy of results, better evaluation of the pollutant concentration spatial distribution and overall better regard for complex terrains. Statistical models, however, have the advantage of providing results quickly and, unlike CFD models, many of them, including the ALOHA 5.4.3 program, have an integrated source term to estimate the extent of the release to the atmosphere as well as a database of physicochemical and toxicological properties of common substances.

As regards application in practice, an important question arises: Which model is more suitable for various land-use planning and crisis management decisionmaking processes? Also, the fact must be taken into account that weather conditions, and thus the size of the thread zone, change significantly with time. Although the objectives of land-use planning and crisis management are varied, modeling of typical pre-agreed scenarios with statistical models should be sufficient for land-use planning. As the potential of CFD modeling is growing, CFD modeling is more suitable for emergency planning, whether with regard to early warning of the public (installation of detectors), recommended behavior of citizens or intervention in case of emergency. It is therefore advisable to gradually move to CFD models in modeling of critical facilities and critical situations involving threats to many citizens.

\section{Conclusion}

The ALOHA 5.4.3 statistical model is not suitable for modeling of accidental releases of gas mixtures in urban areas or complex terrains. The reason is considerable simplification of both inputs and results that can lead to misleading conclusions for these complex conditions. The model generally overestimates the calculation results and presents misleading gas pollutant plume shapes and sizes. It does not consider the influence of mechanical turbulence from surrounding terrain (buildings), it cannot model gas mixtures, the models are only in 2D, and it shows limitations and inaccuracies at low flow velocities or in proximities of pollutant sources. Statistical models of this type are an excellent tool for initial rough estimate of accident consequences for a simple flat terrain and greatly simplified conditions for input data enter. They are not suitable for accurate analyses in urban areas.

The ANSYS Fluent 13.0 numerical CFD model models situations in 3D with a wide range of input data and evaluation tools. It is very suitable for detailed analysis in complex geometries with the option of including a large number of simultaneously acting physical and chemical processes. Thanks to increasing computing power, these types of models are becoming available to researchers who need more accurate results quickly. According to the authors, the numerical models represent a likely future of modeling not only in safety or environmental protection. As regards application in practice, statistical models can be recommended for use in land-use planning even for complex urban environments. It is, however, necessary to achieve a situation where CFD models are used for the purposes of emergency planning and crisis management. 


\section{Acknowledgement}

The article was supported by the Security Research Programme of the Czech Republic in 2010-2015 under the project VG20112013069.

\section{References}

[1] United States Environmental Protection Agency (USEPA). Emergency Management, http://www.epa.gov/osweroe1/content/cameo/aloha.htm

[2] Kozubkova, M., Modeling of Fluid Flow, FLUENT, CFX (in Czech), Ostrava: VSB - Technical University of Ostrava, 153 p., 2008

[3] Bojko M., Guide for Training of Flow Modeling - FLUENT (in Czech), Ostrava: VSB -Technical University of Ostrava, 141 p., 2008

[4] ANSYS Fluent 12.0 Theory Guide - The Contents of This Manual, https:/www.sharcnet.ca/Software/Fluent12/html/ug/node3.htm

[5] Czech Hydrometeorological Institute. Meteorology, http://www.chmi.cz /portal/dt?portal lang=en\&menu=JSPTabContainer/P1_0_Home

[6] Cadastral Bureau of Moravian-Silesian Region. Cadastral Office of Ostrava (in Czech), http://www.nemovitosti-katastr-nahlizeni.cz/katastralni-uradostrava/

[7] Google Earth, http://google-earth.en.softonic.com/

[8] Zelinger, Z., Strizik, M., Kubat, P., Civis, S., Grigorova, E., Janeckova, R., Zavila, O., Nevrly, V., Herecova, L., Bailleux, S., Horka, V., Ferus, M., Skrinsky, J., Kozubkova, M., Drabkova, S., Janour, Z., Dispersion of Light and Heavy Pollutants in Urban Scale Models: $\mathrm{CO}_{2}$ Laser Photoacoustic Studies. Applied spectroscopy, Society for Applied Spectroscopy, pp. 430436, 2009 